\title{
Proyecto de auditoría clínica para el establecimiento y estandarización del Procedimiento de Entrega de Guardia en el Hospital Regional de Alta Especialidad de Ixtapaluca, de septiembre de 2015 a octubre de 2016
}

\author{
Juan José Mario Zaragoza-Saavedra ${ }^{1^{\star}}$, Simón Kawa-Karasik ${ }^{2}$, Alma Rosa Sánchez-Conejo ${ }^{3}$ \\ y Montserrat María del Consuelo Huerta-Hernández ${ }^{4}$ \\ ${ }^{1}$ Subdirector médico C y coordinador de los Servicios de Apoyo al Diagnóstico y Tratamiento, Hospital Regional de Alta Especialidad de Ixtapaluca; ${ }^{2}$ Director general \\ de la Coordinación de los Institutos Nacionales de Salud, Comisión Coordinadora de Institutos Nacionales de Salud y Hospitales de Alta Especialidad; ${ }^{3}$ Directora \\ médica, Hospital Regional de Alta Especialidad de Ixtapaluca; ${ }^{4}$ Asesora de la Dirección General de la Coordinación de los Institutos Nacionales de Salud, Comisión \\ Coordinadora de Institutos Nacionales de Salud y Hospitales de Alta Especialidad
}

\begin{abstract}
RESUMEN
Objetivo: Con el objetivo de mejorar la atención del paciente en nuestro hospital, se implementó la auditoría de la «entrega de guardia». Métodos: El formato de entrega de guardia fue diseñado para evaluar la calidad y la efectividad de la atención del paciente, el intervalo establecido para la implementación de las acciones de mejora en cada turno fue de septiembre de 2015 a octubre de 2016. Resultados: Posterior al periodo de implementación el porcentaje de cumplimiento con estas 5 acciones medidas fueron 1) Interconsulta por un médico especialista, solicitado en el turno por otro personal médico, 98\%; 2) Procedimiento diagnóstico y / o terapéutico solicitado y ejecutado en el mismo turno, 98\%; 3) Ejecución de pendientes entregados y no realizados en el turno previo 97\%; 4) Realización del Formato de Entrega de Guardia 97\%; 5) Adecuado Ilenado del Formato de Entrega de Guardia 100\%. A pesar de tener mejoría significativa la efectividad neta posterior a la implementación de acciones de mejora fue menor del 40\%. Conclusiones: Una vez que estas cinco acciones de mejora fueron implementadas basadas en los procesos de autoevaluación y mejora continua, el porcentaje de cumplimiento de los parámetros evaluados y la eficiencia incrementaron de manera sustancial, en beneficio a la atención de los pacientes del Hospital Regional de Alta Especialidad de Ixtapaluca.
\end{abstract}

Palabras clave: Auditoria Clínica. Entrega de guardia. Calidad.

\section{Correspondencia:}

*Juan José Mario Zaragoza-Saavedra

Hospital Regional de Alta Especialidad de Ixtapaluca

Carretera Federal México Puebla, km 34.5

Pueblo de Zoquiapan

Date of reception: $27-07-2018$

C.P. 56530, Ixtapaluca, Edo. de México

Date of acceptance: 28-08-2018

E-mail: jjmzaragoza@ hotmail.com

Doi: 10.24875/HMCM.18000145 


\section{ABSTRACT}

Background: With the aim of improving patient care at our hospital, a Handoff Audit was carried out. Methods: A Handoff format was designed evaluating the quality and effectiveness of patient care. A time interval was established for the implementation of improvement actions on each work shift, from September 2015 through October 2016. Results: After the implementation period, the percentages of compliance with the 5 actions measured were: 1) interconsultation by a medical specialist, requested on the working day by another physician, 98\%; 2) diagnostic and / or therapeutic procedure requested and executed on the same working day, 98\%; 3) execution of pending tasks not completed in the previous turn, 97\%; 4) compliance with the Handoff Format, 97\%; and 5) adequate completion of the Handoff Format, $100 \%$. Despite the significant progress made after the implementation of improvement actions, the net efficacy was less than 40\%. Conclusions: Once these five actions were implemented based on the self-assessment and continuous improvement processes, the percentage of compliance with the evaluated parameters and efficacy increased substantially, benefiting patient care at the High-Specialty Regional Hospital of Ixtapaluca. (Hosp Med Clin Manag. 2018;11:104-11) Corresponding author: Juan José Mario Zaragoza-Saavedra, jjmzaragoza@ hotmail.com

Key words: Clinical Audit. Handoff. Quality.

\section{INTRODUCCIÓN}

La auditoría médica es un proceso dinámico de mejora continua que permite realizar una evaluación específica de la calidad en la relación médico-paciente, a través de la revisión de los procedimientos creados y destinados por cada hospital para este fin. El objetivo primordial es garantizar que los pacientes atendidos en cualquier institución destinada a salvaguardar la salud tengan garantizada la mejor atención, basándose en la premisa de la calidad total ${ }^{1}$.

En el continente americano, los procesos de auditoría médica tienen su origen en la segunda década del siglo xx. Inicialmente fue un sistema para la acreditación de los hospitales implementado por el Colegio Americano de Cirujanos ${ }^{1}$.

Específicamente, en México los procesos de auditoría médica se originaron en el Instituto Mexicano del Seguro Social, en 1956, y culminaron con la publicación del instructivo «La auditoría médica» de F. González Montesinos en el año $1962^{2}$.

En 1983, ese mismo instituto implementa el Programa de Evaluación Integral de las Unidades Médicas, basado en las teorías de A. Donabedian, quien formulaba que: «la calidad de la atención es aquélla que se espera que pueda proporcionar al usuario el máximo y más completo bienestar después de valorar el balance de ganancias y pérdidas que pueden acompañar al proceso en todas sus partes» ${ }^{3}$.
En 1997, dependiente de la entonces Secretaría de Salubridad y Asistencia, se crea la Dirección de Calidad de la Atención Médica, que a la postre daría lugar a la Dirección General de Calidad y Atención en Salud, que mediante el Sistema Nacional de Indicadores de Calidad en Salud permite registrar, monitorear y dar seguimiento a los indicadores de calidad de los servicios de salud².

En el marco de los procedimientos destinados a la atención del pacientes, en cualquier centro hospitalario se encuentra el pase de visita o entrega de guardia, definido como «un proceso interactivo de comunicación de datos específicos del paciente y de transferencia de responsabilidades, con el propósito de mantener la continuidad de la atención de forma puntual y efectiva en todos los turnos ${ }^{4}$. Si bien la entrega de guardia es un procedimiento común en toda institución, una inadecuada comunicación entre los actores que intervienen en este proceso es referido por parte de la Joint Comission Center for Transforming Healthcare como una de las causas principales de eventos centinela ${ }^{4}$.

La Agencia para la Investigación y Calidad de la Salud y el Consejo de Acreditación de Educación de Graduados en Medicina identifican como prioridad realizar los esfuerzos necesarios para garantizar la seguridad de todos los pacientes ${ }^{5-7}$.

Al respecto, es necesario que las entregas de guardia fomenten una adecuada transmisión de la información y una comunicación eficaz tanto de los médicos 
Tabla 1. IPASS. Mnemotecnia para entrega de guardia ${ }^{4}$

\begin{tabular}{|c|c|c|c|}
\hline I & IIIness severity & Gravedad de la enfermedad & $\begin{array}{l}\text { - Estable } \\
\text { - Inestable (monitorización estricta) }\end{array}$ \\
\hline $\mathrm{P}$ & Patient summary & Resumen del paciente & $\begin{array}{l}\text { - Resumen del estado del paciente } \\
\text { - Principales eventos que lo llevaron al hospital } \\
\text { - Valoración continua } \\
\text { - Plan }\end{array}$ \\
\hline A & Action list & Lista de acciones & $\begin{array}{l}\text { - Pendientes } \\
\text { - Evaluación y liderazgo }\end{array}$ \\
\hline$S$ & Situation awareness and contingency planning & $\begin{array}{l}\text { Situaciones de cuidado, } \\
\text { plan de contingencia }\end{array}$ & $\begin{array}{l}\text { - Saber qué está pasando } \\
\text { - Planear y prevenir lo que puede pasar }\end{array}$ \\
\hline$S$ & Synthesis by receiver & Síntesis del receptor & $\begin{array}{l}\text { - Resumen de lo que el receptor escuchó } \\
\text { - Realizar preguntas } \\
\text { - Reafirmar la lista de pendientes }\end{array}$ \\
\hline
\end{tabular}

adscritos como de los médicos en formación, minimizando al máximo el riesgo de presentar algún evento de falla, cuasifalla o centinela. Es de particular importancia la transmisión adecuada de la información en la entrega de guardia de los pacientes hospitalizados en el área de urgencias, así como la optimización del tiempo para este procedimiento médico administrativo, por lo que la implementación de un instrumento y un procedimiento para la entrega de pacientes y pendientes en estas áreas ha demostrado ser un control adecuado en la calidad de la atención ${ }^{6}$.

Con el fin de abatir los defectos de comunicación y posibles fallos en el tratamiento de pacientes hospitalizados, se han diseñado y propuesto diferentes métodos para la entrega de guardia, en los cuales figura el procedimiento denominado mnemotécnicamente como «IPASS», por sus siglas en inglés, que se refiere a cinco pasos a seguir en la entrega de cualquier paciente de un médico a otro, haciendo referencia a: (I - IIness) la gravedad de la enfermedad, ( $\mathrm{P}$ - Pacient) resumen del paciente, (A - Action list) lista de acciones, (S - Situation) conciencia de la situación y planificación, y (S - Synthesis by receiver) síntesis del receptor ${ }^{8,9}$, con mayor desglose en la tabla 1.

Partiendo de este procedimiento, el Hospital Regional de Alta Especialidad de Ixtapaluca implementó su propio sistema de entrega de guardia mediante un formato en una tabla de cálculo que puede llenarse en tiempo real y en cualquier dispositivo portátil, y al mismo tiempo permite la transmisión masiva a todo el personal operativo, agilizando así la atención y la calidad por medio de este formato, y aportando la estructura, el soporte y los elementos necesarios para la toma de decisiones inmediatas en beneficio de los pacientes.

En función de la implementación de este procedimiento y formato se decidió evaluar previamente mediante un proceso de mejora continua que pudiera establecer las acciones de mejora y otorgar una atención de calidad y eficiente, por lo que se realizó una auditoría clínica como herramienta para la localización de áreas de oportunidad, en la cual se detectaron problemas de comunicación entre el personal médico, y rezago en la realización de interconsultas, procedimientos diagnósticos y terapéutica de los pacientes hospitalizados en nuestra institución.

\section{MATERIAL Y MÉTODOS}

En el presente trabajo de auditoría médica, y con base en la identificación de los posibles riesgos, se buscó crear un instrumento de comunicación interno efectivo para el personal que integra los seis turnos asignados a la Dirección Médica del Hospital Regional de Alta Especialidad de Ixtapaluca, y al mismo tiempo estandarizar el procedimiento de entrega de guardia, garantizando así la posibilidad de disminuir los eventos adversos, eventos centinela y cuasifallas, mediante la realización de acciones preventivas y correctivas que impliquen por sí mismas el cuidado efectivo de los pacientes atendidos en los diferentes servicios del nuestra institución.

La labor de la auditoría clínica para este procedimiento de entrega de guardia se llevó a cabo del 18 de 


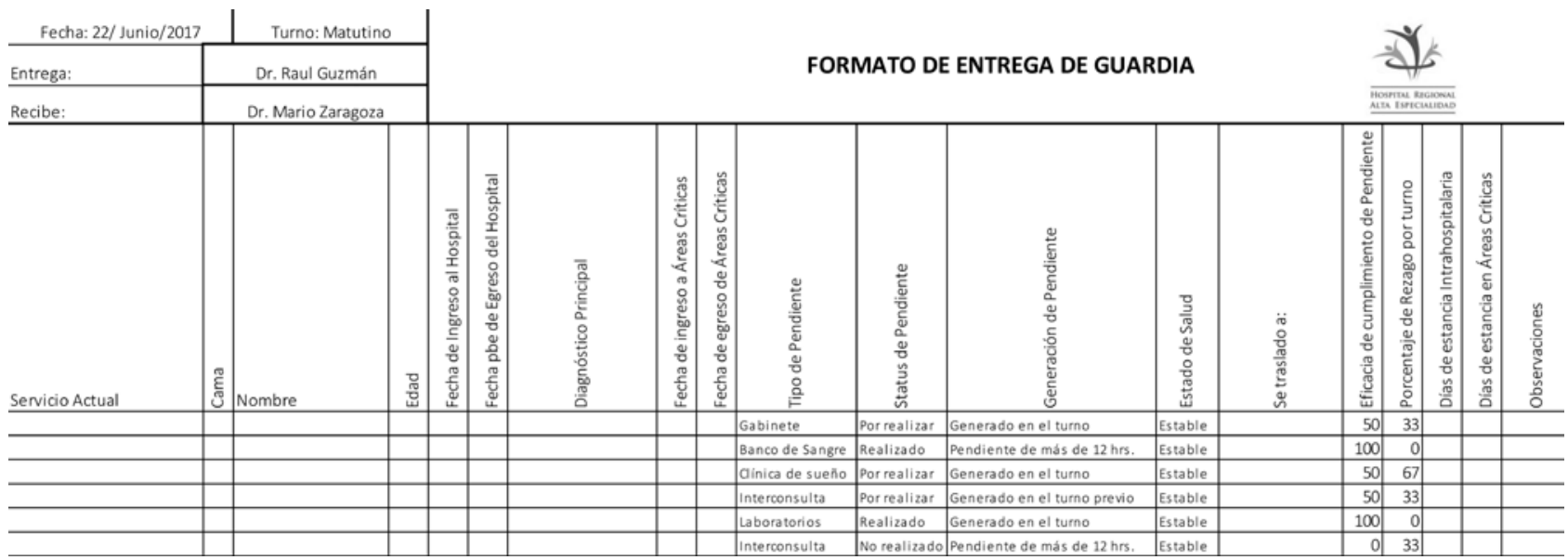

Figura 1. Formato de entrega de guardia implementado para el procedimiento de pase de visita en el Hospital Regional de Alta Especialidad de Ixtapaluca.

septiembre de 2015 al 18 de octubre de 2016, estableciendo como fecha de corte para la evaluación e implementación de las acciones de mejora el 20 de noviembre de 2015. El análisis de los resultados obtenidos en seguimiento a las propuestas de mejora y a las recomendaciones hechas en la fecha de corte fue realizado el 18 de octubre de 2016. Los resultados fueron presentados en el Primer Congreso de los Hospitales Regionales de Alta Especialidad, en la Ciudad de León (Guanajuato), en noviembre de 2016.

De primera instancia se estableció y autorizó el formato de entrega de guardia en agosto de 2015, utilizando para ello una hoja de cálculo Excel del programa de paquetería de Microsoft Office $2010^{\circledR}$. La planeación de este formato se enfocó en la recolección de datos específicos que ayuden a la toma de decisiones para la atención de los pacientes ingresados en las diferentes áreas de hospitalización, urgencias, servicios ambulatorios y quirófanos del Hospital Regional de Alta Especialidad de Ixtapaluca, con especial énfasis en la comunicación efectiva en cuanto a los pendientes para cada turno. Se anexa imagen del formato (Fig. 1).

La cumplimentación y la aplicación de este formato de entrega de guardia fueron encomendadas a los asistentes de la dirección de cada turno, con el soporte de los médicos de guardia de las diferentes áreas donde se encontrara ingresado algún paciente que requiriera algún estudio de laboratorio, estudio de gabinete, procedimiento diagnóstico o procedimiento terapéutico para su seguimiento.
Durante esta implementación se definieron de forma conjunta los parámetros a evaluar. Se seleccionaron cinco parámetros según las necesidades del hospital y las áreas de oportunidad y mejora. Los parámetros a valorar fueron: a) interconsulta ejecutada por un médico especialista solicitada en el turno por otro personal médico, b) procedimiento diagnóstico y/o terapéutico solicitado y ejecutado en el mismo turno, c) ejecución de pendientes entregados y no realizados en el turno previo, d) realización y estandarización del formato de entrega de guardia, y e) adecuada cumplimentación del formato de entrega de guardia.

Una vez aprobado el formato y definidos los parámetros a analizar dentro de la auditoría médica, se realizó una evaluación previa capacitación para poder determinar los puntos de mayor énfasis dentro de la enseñanza, tomando como base el modelo descrito en el trabajo publicado por A. Starmer en 2014, por lo que se capacitó a cada uno de los asistentes de la dirección y jefes de área en una entrega de guardia de calidad y con las herramientas necesarias para mejorar la seguridad del paciente, y se adiestró en la cumplimentación y ejecución del formato de entrega de guardia durante una sesión única de 90 min de duración. Posterior a esta capacitación, nuevamente se realizó una evaluación para determinar el nivel de impacto y de conocimientos adquiridos.

La muestra total a analizar de acuerdo con el número de entregas de guardia durante el periodo de estudio establecido fue de 416, los cuales se obtuvieron aleatoriamente de cada uno de los seis turnos que integran las jornadas laborales del hospital, divididas en dos 
Tabla 2. Tablero de parámetros y de establecimiento de metas para el proceso de entrega de guardia, establecido en agosto de 2015

Evidencia de calidad

Interconsulta de valoración ejecutada por el especialista

solicitada en el turno por otro personal médico

Procedimiento diagnóstico y/o terapéutico solicitado y

ejecutado en el mismo turno

Ejecución de pendientes entregados y no realizados en el turno previo

Realización del formato de entrega de guardia

Cumplimentación adecuada del formato de entrega

de guardia

\section{Porcentaje de} cumplimiento esperado

$\begin{array}{lc}100 \% & 0 \% \\ 100 \% & 0 \% \\ 100 \% & 0 \% \\ 100 \% & 0 \% \\ 100 \% & 0 \%\end{array}$

\section{Excepción}

$0 \%$

$\%$

$\%$

$0 \%$

$0 \%$ ciclos. El primer ciclo consta de 130 formatos y el segundo de 286.

La evaluación de los formatos en ambos ciclos corrió a cargo de la Dirección Médica y de las tres subdirecciones que la integran (especialidades médico-clínicas, especialidades médico-quirúrgicas y de soporte al diagnóstico y tratamiento), para la implementación de acciones de mejora y emisión de recomendaciones finales para ser aplicadas al final de la auditoría.

La dinámica de entrega de guardia se realizó basándose en el modelo IPASS y en el formato que se realizó previamente en el que los jefes de área entregaban la guardia al asistente de la dirección del turno vespertino, y éste, a su vez, hacía lo propio con los asistentes de los turnos nocturnos, los cuales se enlazaban con los turnos matutino y jornadas especiales matutinas, cerrando el ciclo con la jornada especial nocturna. Bajo este proceso se previó la generación de 19 reportes semanales (uno por cada entrega de guardia), que incluyen: cinco reportes del turno matutino, cinco reportes del turno vespertino, siete reportes de los turnos nocturnos y dos reportes de la jornada especial matutina.

Se estableció como meta que el cumplimiento de los cinco parámetros a auditar fuera de un ciento por ciento, y esperando una efectividad también del ciento por ciento, sin margen de tolerancia.

\section{RESULTADOS}

Una vez establecidos los parámetros a auditar y determinados el total de reportes a entregar, se diseñó un tablero de metas con el porcentaje a alcanzar para el personal de asistentes de dirección y jefes de área (Tabla 2).

Los resultados obtenidos durante la primera etapa de la auditoría de los cinco parámetros seleccionados se reportan en la tabla 3 .

Para el cierre de la primera etapa fueron seleccionados 130 formatos de manera aleatoria, integrados por las seis jornadas de trabajo con las que cuenta el Hospital Regional de Alta Especialidad de Ixtapaluca.

En esta evaluación quedó evidenciado que un elevado porcentaje de procedimientos diagnósticos y/o terapéuticos no eran realizados durante el turno en el cual se solicitaban, con un $80 \%$ de diferimiento al siguiente turno, ocasionando retraso en el tratamiento de los pacientes al menos de $8 \mathrm{~h}$. Este escenario incrementaba la carga de trabajo de manera exponencial a los turnos matutino y matutino de jornada acumulada, aunado ello a un retraso en la ejecución de interconsultas del 65\%.

Los resultados obtenidos en esta primera etapa permitieron evidenciar un porcentaje de efectividad del $42.8 \%$ en lo referente al rendimiento del personal médico, y basándose en ello fue posible proponer cinco acciones de mejora para ejecutar en la segunda etapa de la auditoría, las cuales se muestran en la tabla 4, para ser realizadas de manera inmediata y permanente.

Una vez analizadas y avaladas las propuestas de mejora y las recomendaciones, se dieron a conocer al personal asignado como asistentes de dirección y jefes de área para la puesta en marcha de manera inmediata 
Tabla 3. Resultados de los porcentajes obtenidos de la evaluación individual de cada parámetro en la primera etapa de la auditoría, con un total de 130 formatos seleccionados

\begin{tabular}{lccc}
\hline Evidencia de calidad & $\begin{array}{c}\text { Formatos } \\
\text { evaluados }\end{array}$ & $\begin{array}{c}\text { Parámetro cumplido } \\
\text { al 100\% }\end{array}$ & $\begin{array}{c}\text { Porcentaje } \\
\text { deumplimiento }\end{array}$ \\
\hline $\begin{array}{l}\text { Interconsulta de valoración ejecutada por el especialista } \\
\text { solicitada en el turno por otro personal médico }\end{array}$ & 130 & 46 & 35 \\
$\begin{array}{l}\text { Procedimiento diagnóstico y/o terapéutico solicitado y } \\
\text { ejecutado en el mismo turno }\end{array}$ & 130 & 26 & 20 \\
$\begin{array}{l}\text { Ejecución de pendientes entregados y no realizados en } \\
\text { el turno previo }\end{array}$ & 130 & 104 & 35 \\
$\begin{array}{l}\text { Realización del formato de entrega de guardia } \\
\text { Cumplimentación adecuada del formato de entrega de } \\
\text { guardia }\end{array}$ & 130 & 68 & 27 \\
\hline
\end{tabular}

Tabla 4. Propuestas de mejora y recomendaciones posteriores al término de la primera etapa de la implementación del formato de entrega de guardia

\begin{tabular}{|c|c|c|}
\hline Propuestas de mejora y recomendaciones & Responsable & Inicio y vigencia \\
\hline $\begin{array}{l}\text { Seguimiento específico de las interconsultas generadas } \\
\text { por turno, verificando el procedimiento de entrega y } \\
\text { recepción según el manual de procedimientos. } \\
\text { Realización de verificación de nota de valoración y plan } \\
\text { terapéutico propuesto por el especialista } \\
\text { interconsultado asentado en el expediente }\end{array}$ & $\begin{array}{l}\text { Asistente de dirección } \\
\text { y jefes de área }\end{array}$ & Inmediato y permanente \\
\hline $\begin{array}{l}\text { Verificación de la ejecución de los procedimientos } \\
\text { diagnósticos y terapéuticos generados en cada turno, } \\
\text { mediante la revisión de la solicitud de procedimientos } \\
\text { solicitados en el expediente electrónico al inicio y final } \\
\text { de cada turno }\end{array}$ & $\begin{array}{l}\text { Asistente de dirección } \\
\text { y jefes de área }\end{array}$ & Inmediato y permanente \\
\hline $\begin{array}{l}\text { Priorizar en el formato de entrega de guardia los } \\
\text { procedimientos pendientes que no se realizaron en } \\
\text { el turno que concluye, indicando en el rubro de } \\
\text { observaciones los motivos por los cuales no se } \\
\text { ejecutaron para la resolución inmediata en el turno } \\
\text { siguiente }\end{array}$ & $\begin{array}{l}\text { Asistente de dirección } \\
\text { y jefes de área }\end{array}$ & Inmediato y permanente \\
\hline $\begin{array}{l}\text { Firma física del formato de entrega de guardia por parte } \\
\text { del personal que entrega el turno y del que lo recibe }\end{array}$ & $\begin{array}{l}\text { Asistente de dirección } \\
\text { y jefes de área }\end{array}$ & Inmediato y permanente \\
\hline $\begin{array}{l}\text { Envío del formato de entrega de guardia en versión } \\
\text { electrónica, y entrega del original a la dirección médica } \\
\text { y de copias simples a las subdirecciones médica, } \\
\text { quirúrgica y de soporte al diagnóstico y tratamiento }\end{array}$ & $\begin{array}{l}\text { Asistente de dirección } \\
\text { y jefes de área }\end{array}$ & Inmediato y permanente \\
\hline
\end{tabular}

y permanente a partir del 22 de noviembre de 2015, plantado como fecha de corte y evaluación para la entrega de los resultados el 18 de octubre de 2016.

En esta segunda etapa se analizaron un total de 286 formatos basados en los cinco parámetros establecidos al inicio del proceso de auditoría, que arrojó los resultados vertidos en la tabla 5.
Al término de la segunda etapa del proceso de auditoría de entrega de guardia se evidenció de manera radical una mejora en el cumplimiento de los parámetros establecidos, alcanzando el $97.5 \%$ de efectividad del proceso de entrega de guardia. Las propuestas emitidas permitieron dar cumplimiento prácticamente a todas las solicitudes de valoración y realización de procedimientos diagnósticos y/o terapéuticos en la misma 
Tabla 5. Resultados de los porcentajes obtenidos de la evaluación individual de cada parámetro en la segunda etapa de la auditoría, con un total de 286 formatos seleccionados

\begin{tabular}{lccc}
\hline Evidencia de calidad & $\begin{array}{c}\text { Formatos } \\
\text { evaluados }\end{array}$ & $\begin{array}{c}\text { Parámetro cumplido } \\
\text { al 100\% }\end{array}$ & $\begin{array}{c}\text { Porcentaje } \\
\text { de cumplimiento }\end{array}$ \\
\hline $\begin{array}{l}\text { Interconsulta de valoración ejecutada por el } \\
\text { especialista solicitada en el turno por otro } \\
\text { personal médico }\end{array}$ & 286 & 280 & 98 \\
$\begin{array}{l}\text { Procedimiento diagnóstico y/o terapéutico } \\
\text { solicitado y ejecutado en el mismo turno }\end{array}$ & 286 & 280 & 98 \\
$\begin{array}{l}\text { Ejecución de pendientes entregados y no } \\
\text { realizados en el turno previo }\end{array}$ & 286 & 277 & 97 \\
$\begin{array}{l}\text { Realización del formato de entrega de guardia } \\
\text { Cumplimentación adecuada del formato de } \\
\text { entrega de guardia }\end{array}$ & 286 & 275 & 96 \\
\hline
\end{tabular}

Tabla 6. Resultados y porcentajes finales de efectividad de los parámetros establecidos para el proceso de auditoría de entrega de guardia

\begin{tabular}{lcccc}
\hline Evidencia de calidad & $\begin{array}{c}\text { Formatos } \\
\text { evaluados }\end{array}$ & $\begin{array}{c}\text { Porcentaje de } \\
\text { cumplimiento } \\
\text { primera etapa }\end{array}$ & $\begin{array}{c}\text { Porcentaje de } \\
\text { cumplimiento } \\
\text { segunda etapa }\end{array}$ & $\begin{array}{c}\text { Porcentaje de } \\
\text { efectividad real }\end{array}$ \\
\hline $\begin{array}{l}\text { Interconsulta de valoración ejecutada por el } \\
\text { especialista solicitada en el turno por otro } \\
\text { personal médico }\end{array}$ & 416 & 35 & 98 \\
$\begin{array}{l}\text { Procedimiento diagnóstico y/o terapéutico } \\
\text { solicitado y ejecutado en el mismo turno }\end{array}$ & 416 & 20 & 98 \\
$\begin{array}{l}\text { Ejecución de pendientes entregados y no } \\
\text { realizados en el turno previo }\end{array}$ & 416 & 80 & 97 \\
$\begin{array}{l}\text { Realización del formato de entrega de guardia } \\
\text { Cumplimentación adecuada del formato de } \\
\text { entrega de guardia }\end{array}$ & 416 & 27 & 96 \\
\hline
\end{tabular}

jornada de trabajo en las que fueron generadas. La correcta cumplimentación del formato de entrega de guardia alcanzó un 100\% de efectividad, disminuyendo la posibilidad de presentarse algún evento de falla o cuasifalla, y logrando evidenciar de manera inmediata algún evento centinela.

Sin embargo, y a pesar de la inminente mejoría en la eficiencia del procedimiento de entrega de guardia en el Hospital Regional de Alta Especialidad de Ixtapaluca, se decidió realizar un promedio de cumplimiento real de los parámetros establecidos, los cuales se aprecian mejor en la tabla 6 .

Una vez analizados los resultados de ambas etapas y tras compararlas, se dedujo que existe una mejoría evidente y exponencial en los cinco parámetros establecidos en la auditoría.

Aunque cabe señalar el hecho de que la efectividad real de los procedimientos de entrega de guardia fue del $39.8 \%$, que es el promedio de los resultados reales de efectividad de la quinta columna de la tabla 6. Queda claro el avance en la dinámica de las actividades del personal médico encargado de la atención de los pacientes de nuestra institución y del seguimiento que se hizo por parte de los asistentes de dirección y jefes área, alcanzando una resolución total de procedimientos y valoraciones por turno del $98 \%$ en ambos parámetros, que de manera simple puede traducirse como la resolución de 9.8 pendientes de cada 10 generados en una jornada laboral.

\section{CONCLUSIONES}

Para finalizar, es necesario especificar que, bajo los resultados obtenidos en la primera etapa, sería imposible llegar a una evaluación final del 100\% de efectividad real, para lo cual los resultados de cumplimiento tendrían que haber sido perfectos desde la primera evaluación y, en consecuencia, no hubiera dado pie al 
proceso de autoría, ya que no existirían acciones de mejora a implementar.

Es importante recalcar que el trabajo conjunto por parte de la institución logró que se llevara a cabo esta auditoría clínica involucrando a todo el personal, por una mejor atención de calidad y seguridad para nuestros pacientes.

\section{BIBLIOGRAFÍA}

1. Solar MA. Auditoría médica I. Rev Med Chile. 1960;88:499.

2. Instituto Mexicano del Seguro Social; Subdirección General Médica; Depar tamento de Planeación Técnica de los Servicios Médicos. La auditoría médica. 1. ${ }^{a}$ ed. México: IMSS; 1962.
3. Donabedian A. The definition of quality and aproaches to its assessment. En: Explorations in quality assessment and monitoring. Vol. I. Ann Arbor, Michigan: Health Administration Press; 1980.

4. Starmer AJ, Spector ND, Srivastava R, Allen AD, Landrigan CP, Sectish TC I-PASS Study Group. I-pass, a mnemonic to standardize verbal handoffs. Pediatrics. 2012;129(2);201-4.

5. Improving Transitions in Care: Handoff Communications. Joint Commission Center for Transforming Healthcare Web site. Disponible en: http://www. centerfortransforminghealthcare.org/assets/4/6/handoff_comm_storyboard. pdf.

6. McDonald K. Closing the Quality Gap: A Critical Analysis of Quality Improvement Strategies. Vol 7. Rockville, MD: Care Coordination; 2007.

7. Nasca TJ, Day SH, Amis ES Jr; ACGME Duty Hour Task Force. The new recommendations on duty hours from the ACGME Task Force. N Engl J Med. 2010;363(2):e3.

8. Rüdiger-Stürchler M, Keller DI, Bingisser R. Emergency physician intershift handover - can a dINAMO checklist speed it up and improve quality? Swiss Med Wkly. 2010;24:140:w13085.

9. Starmer AJ, Sectish TC, Simon DW, Keohane C, McSweeney ME, Chung EY. Changes in Medical Errors after Implementation of a Handoff Program. N Engl J Med. 2014;371(19):1803-12. 